\title{
The CEV Model and Its Application in a Study of Optimal Investment Strategy
}

\author{
Aiyin Wang, ${ }^{1,2}$ Ls Yong, ${ }^{1}$ Yang Wang, ${ }^{1}$ and Xuanjun Luo ${ }^{1}$ \\ ${ }^{1}$ The School of Finance, Southwestern University of Finance and Economics, Chengdu 610074, China \\ ${ }^{2}$ Department of Mathematics, Xinjiang University of Finance and Economics, Urumqi 830012, China \\ Correspondence should be addressed to Aiyin Wang; way8848@sina.com
}

Received 2 June 2014; Revised 7 July 2014; Accepted 22 July 2014; Published 13 August 2014

Academic Editor: Shouming Zhong

Copyright (c) 2014 Aiyin Wang et al. This is an open access article distributed under the Creative Commons Attribution License, which permits unrestricted use, distribution, and reproduction in any medium, provided the original work is properly cited.

\begin{abstract}
The constant elasticity of variance $(C E V)$ model is used to describe the price of the risky asset. Maximizing the expected utility relating to the Hamilton-Jacobi-Bellman $(H J B)$ equation which describes the optimal investment strategies, we obtain a partial differential equation. Applying the Legendre transform, we transform the equation into a dual problem and obtain an approximation solution and an optimal investment strategies for the exponential utility function.
\end{abstract}

\section{Introduction}

The constant elasticity of variance $(C E V)$ model is a natural extension of the geometric Brownian motion $(G B M)$. The Merton's model and its extensions [1-3] were generally studied under the assumption that risky asset price was described by the GBM. The $C E V$ model is originally proposed by Cox and Ross [4] as an alternative diffusion process for European option pricing [5-8]. Comparing with the GBM, we know that the advantages of the $C E V$ model are that the volatility rate has correlation with the risky asset price and can explain the empirical bias such as volatility smile [9]. The $C E V$ model was usually applied to calculate the theoretical price, sensitivities, and implied volatility of options [1012]. In recent years, the optimal investment problem for a pension fund has become an important subject [13-17], in which the $C E V$ model was applied to study the optimal investment strategy for a defined contribution $(D C)$ pension plan. However, the application of the $C E V$ model to the investment and consumption problem has not been widely reported in the existing academic articles.

In this paper, we introduce the $C E V$ model into an investment and consumption problem and optimally allocate the wealth between one risk-free asset and one risky asset. We use $C E V$ model to describe the risk asset's price and discuss personal optimal portfolio with consumption under the framework of the classical Merton's portfolio optimization problems. Our goal is to choose an optimal investment strategy to maximize total expected utility of wealth. By using the method of stochastic optimal control, the corresponding Hamilton-Jacobi-Bellman ( $H J B)$ equation for the value function of the optimization problem is obtained. Applying the Legendre transform and dual theory $[14,15]$, we obtain an approximation solution and the optimal investment strategies for the exponential utility function. The novelty of this paper is different from those of [13-17], in which the CEV model is used to study the optimal investment strategy for pension fund, we use $C E V$ model to study personal optimal portfolio with consumption factors.

This paper is organized as follows. We introduce the CEV model about the personal portfolio in Section 2. In Section 3, we turn our stochastic optimal problem into a corresponding Hamilton-Jacobi-Bellman $(H J B)$ equation. We transform our problem into the dual problem by applying the Legendre transform and the dual theory in Section 4. In Section 5, we derive an appriximation solution by choosing the CARA utility. Conclusions are given in Section 6.

\section{The $C E V$ Model}

In this section, we consider that the market structure consists of a risk-free asset and a single risky asset which is described 
by the CEV model. With personal consumption, the dynamical equation of personal portfolio is established.

We denote the price of the risk-free asset (i.e., the bank account) at time $t$ by $B_{t}$, which satisfies the following formula:

$$
d B_{t}=r B_{t} d t
$$

where $r$ is a constant rate of interest.

We denote the price of the risky asset (hereinafter called "stock") at time $t$ by $S_{t}$, which satisfies the $C E V$ model. Consider

$$
\frac{d S_{t}}{S_{t}}=\mu d t+k S_{t}^{\beta} d W_{t}
$$

where $\mu$ is an expected instantaneous return of the stock and satisfies the general condition $\mu>\beta . k S_{t}^{\beta}$ is the instantaneous volatility and $\beta$ is the elasticity parameter and satisfies the general condition $\beta<0$. $W_{t}: t \geq 0$ is a standard Brownian motion defined on a complete probability space $(\Omega, \mathscr{F}, P)$, where $P$ is the risk-neutral probability. The filtration $\mathscr{F}=$ $\left\{\mathscr{F}_{t}\right\}$ is a right continuous filtration of sigma-algebras on this space.

For personal portfolio, we denote personal consumption at time $t$ by $\lambda_{t}$ which is described by an arithmetic Brownian motion as follows:

$$
d \lambda_{t}=b d t+a d M_{t}
$$

where $M_{t}$ is a standard Brownian motion, $a$ and $b$ are constants. Generally, $b$ is written as a function of $t$ and $\lambda_{t}$, that is, $b=b\left(\lambda_{t}, t\right)$, and $a$ is written as a function of $t$. Here, for simplicity, $a$ and $b$ are regarded as constants. Assume that the Brownian motion $W_{t}$ is correlated with $M_{t}$ and the correlation coefficient is $\rho$, that is, $d W_{t} d M_{t}=\rho$.

Let $V_{t}$ denote one's disposable wealth at time $t \in[0, T]$, $\pi_{t}$ and let $1-\pi_{t}$ denote the proportion of one's disposable wealth $V_{t}$ invested in the risky asset and the risk-free asset, respectively. The dynamical equation of personal portfolio is given by

$$
d V_{t}=\pi_{t} V_{t}\left(\mu d t+k S_{t}^{\beta} d W_{t}\right)+\left(1-\pi_{t}\right) V_{t} r d t-\lambda_{t} d t .
$$

To seek optimal investment strategy $\pi_{t}$, we maximize the expected utility of the terminal wealth

$$
\max _{\pi_{t}} E\left\{U\left(V_{T}\right) \mid \mathscr{F}_{t}\right\}
$$

where $U(\cdot)$ is increasing and concave.

\section{The Hamilton-Jacobi-Bellman Equation}

In this section, we obtain the form of optimal investment strategy $\pi_{t}$ and the Hamilton-Jacobi-Bellman equation of the optimal problem (5) by using the dynamic programming approach.

Define the value function for the optimal problem (5) as

$$
H\left(t, S_{t}, V_{t}, \lambda_{t}\right)=\sup _{\pi_{t} \in \mathscr{A}} E\left\{U\left(V_{T}\right) \mid \mathscr{F}_{t}\right\}, \quad 0<t<T,
$$

where $\mathscr{A}$ is the set of admissible trading strategies. Then, the wealth process becomes Markov process. Consider

$$
d V_{t}=\left(\pi_{t} V_{t} \mu+\left(1-\pi_{t}\right) r V_{t}-\lambda_{t}\right) d t+\pi_{t} V_{t} k S_{t}^{\beta} d W_{t}
$$

We define the value function

$$
\begin{aligned}
H^{\pi}\left(t, S_{t}, V_{t}, \lambda_{t}\right) & =E\left\{U\left(V_{T}^{\pi}\right) \mid \mathscr{F}_{t}\right\} \\
& =E\left\{U\left(V_{T}^{\pi}\right) \mid S_{t}=s, V_{t}=v, \lambda_{t}=\lambda\right\} .
\end{aligned}
$$

Using the Markov property and the law of iterated expectation, we know that $H^{\pi}(t, s, v, \lambda)$ is a martingale. The value function $H(t, s, v, \lambda)=\sup _{\pi_{t} \in \mathscr{A}} H^{\pi}(t, s, v, \lambda)$ is also a martingale.

Using Itŏ formula, we have

$$
\begin{gathered}
H\left(t, S_{t}, V_{t}, \lambda_{t}\right) \\
=\sup _{\pi_{t} \in \mathscr{A}} E_{t}\left\{H\left(t+\Delta t, S_{t+\Delta t}, V_{t+\Delta t}, \lambda_{t+\Delta t}\right)\right\} \\
=\sup _{\pi_{t} \in \mathscr{A}} E_{t}\left\{H\left(t, S_{t}, V_{t}, \lambda_{t}\right)+H_{S_{t}} d S_{t}\right. \\
+H_{\lambda_{t}} d \lambda_{t}+\frac{1}{2} H_{S_{t} S_{t}} d S_{t} d S_{t} \\
+\frac{1}{2} H_{V_{t} V_{t}} d V_{t} d V_{t}+\frac{1}{2} H_{\lambda_{t} \lambda_{t}} d \lambda_{t} d \lambda_{t} \\
+H_{S_{t} V_{t}} d S_{t} d V_{t}+H_{\lambda_{t} V_{t}} d \lambda_{t} d V_{t} \\
\left.+H_{S_{t} \lambda_{t}} d S_{t} d \lambda_{t}\right\} .
\end{gathered}
$$

Considering the equation $H(T, s, v, \lambda)=U\left(V_{T}\right)$, we have the following Hamilton-Jacobi-Bellman $(H J B)$ equation associated with the optimization problem

$$
\begin{aligned}
& H_{t}+\mu s H_{s}+(r v-\lambda) H_{v}+b H_{\lambda}+\frac{1}{2} k^{2} s^{2 \beta+2} H_{s s} \\
& +\frac{1}{2} a^{2} H_{v v}-a k s^{\beta+1} \rho H_{s v} \\
& +\sup _{[\pi]}\left\{\frac{1}{2} \pi^{2} v^{2} k^{2} s^{2 \beta} H_{v v}+\pi v(\mu-r) H_{v}\right. \\
& \left.\quad+\pi v k^{2} s^{2 \beta+1} H_{s v}+\pi v a \rho k s^{\beta} H_{v \lambda}\right\}=0,
\end{aligned}
$$

where $H_{t}, H_{s}, H_{v}, H_{\lambda}, H_{s s}, H_{\lambda \lambda}, H_{v v}, H_{s v}, H_{v \lambda}$, and $H_{s \lambda}$ denote partial derivatives of first and second orders with respect to time, stock price, wealth, and consumption.

Differentiating (10) with respect to $\pi$, we have

$$
\begin{aligned}
& \pi v^{2} k^{2} s^{2 \beta} H_{v v}+v(\mu-r) H_{v} \\
& \quad+v k^{2} s^{2 \beta+1} H_{s v}+v a \rho k s^{\beta} H_{v \lambda}=0 .
\end{aligned}
$$

The optimal strategy $\pi_{t}^{*}$ is

$$
\pi_{t}^{*}=-\frac{(\mu-r) H_{v}+k^{2} s^{2 \beta+1} H_{s v}+a \rho k s^{\beta} H_{v \lambda}}{v k^{2} s^{2 \beta} H_{v v}} .
$$


Putting (12) into (10), we obtain a partial differential equation for the value function $H$

$$
\begin{aligned}
H_{t} & +\mu s H_{s}+(r v-\lambda) H_{v}+b H_{\lambda}+\frac{1}{2} k^{2} s^{2 \beta+2} H_{s s} \\
& +\frac{1}{2} a^{2} H_{\lambda \lambda}+a \rho k s^{\beta+1} H_{s \lambda} \\
& -\frac{\left[(\mu-r) H_{v}+k^{2} s^{2 \beta+1} H_{s v}+a \rho k s^{\beta} H_{v \lambda}\right]^{2}}{2 k^{2} s^{2 \beta} H_{v v}}=0 .
\end{aligned}
$$

\section{The Legendre Transform and Dual Theory}

Definition 1. Let $f: R^{n} \rightarrow R$ be a convex function. For $z>0$, we write the Legendre transform

$$
L(z)=\max _{x}\{f(x)-z x\} .
$$

The function $L(z)$ is called the Legendre dual of the function $f(x)$ (see [16]).

If $f(x)$ is strictly convex, the maximum in the above equation will be attained at just one point, which is denoted by $x_{0}$. It arrives at the unique solution to the first-order condition, namely,

$$
\frac{d f(x)}{d x}-z=0
$$

Therefore, we write

$$
L(z)=f\left(x_{0}\right)-z x_{0} .
$$

According to Definition 1, we take advantage of the convexity of value function $H(t, s, \lambda, v)$ to define the Legendre transform

$$
\begin{array}{r}
\widehat{H}(t, s, \lambda, z):=\sup _{v>0}\{H(t, s, \lambda, v)-z v \mid 0<v<\infty\}, \\
(0<t<T),
\end{array}
$$

where $z>0$ denotes the dual variable to $v$. The value of $v$ where this optimum is attained is denoted by $g(t, s, \lambda, z)$. Namely,

$$
\begin{array}{r}
g(t, s, \lambda, z):=\inf _{v>0}\{v \mid H(t, s, \lambda, v) \geq z v+\widehat{H}(t, s, \lambda, z)\} \\
(0<t<T) .
\end{array}
$$

Using (16) and (17), we have

$$
z=H_{v} .
$$

From (18) and (4), the function $\widehat{H}$ related to $g$ is given by $g=$ $-\widehat{H}_{z}$. Therefore, we take one of the two functions $g$ and $\widehat{H}$ as the dual of $H$. Here, we work mainly with the function $g$, as it is easy to be computed numerically for the purpose of computing optimal strategies.

Differentiating (18) and (4) with respect to $t, s$, and $z$, we know that the transformation rules for the derivatives of the value function $H$ and the dual function $\widehat{H}$ are given by $($ see $[13,16])$

$$
\begin{gathered}
\widehat{H}(t, s, \lambda, z)=H(t, s, \lambda, g)-z g, \quad g(t, s, \lambda, z)=v,(20) \\
H_{v}=z, \quad H_{t}=\widehat{H}_{t}, \quad H_{s}=\widehat{H}_{s}, \quad H_{\lambda}=\widehat{H}_{\lambda}, \\
g=-\widehat{H}_{z}, \quad H_{v v}=-\frac{1}{\widehat{H}_{z z}}, \quad H_{s s}=\widehat{H}_{s s}-\frac{\widehat{H}_{s z}}{\widehat{H}_{z z}}, \\
H_{\lambda \lambda}=\widehat{H}_{\lambda \lambda}-\frac{\widehat{H}_{z \lambda}^{2}}{\widehat{H}_{z z}}, \quad H_{v s}=-\frac{\widehat{H}_{s z}}{\widehat{H}_{z z}}, \quad H_{v \lambda}=-\frac{\widehat{H}_{\lambda z}}{\widehat{H}_{z z}}, \\
H_{s \lambda}=\widehat{H}_{s \lambda}-\frac{\widehat{H}_{s z} \widehat{H}_{z \lambda}}{\widehat{H}_{z z}} .
\end{gathered}
$$

At the terminal time $T$, we define

$$
\begin{gathered}
\widehat{U}(z)=\sup _{v>0}\{U(v)-z v\}, \\
G(z)=\inf _{v>0}\{v \mid U(v) \geq z v+\widehat{U}(z)\} .
\end{gathered}
$$

Kramkov and Schachermayer [18] and Cox and Huang [19] have shown that the functions $\widehat{U}(z)$ and $U(v)$ can be obtained from each other by using the Legendre transform as follows:

$$
\widehat{U}(z)=\sup _{v>0}\{U(v)-z v\}, \quad U(v)=\inf _{v>0}\{\widehat{U}(z)+z v\} .
$$

Thus, the primary problem is turned into a dual problem.

Putting (21) into (13) yields

$$
\begin{aligned}
\widehat{H}_{t} & +\mu s \widehat{H}_{s}+(r v-\lambda) z+b \widehat{H}_{\lambda}+\frac{1}{2} k^{2} s^{2 \beta+2} \widehat{H}_{s s}+\frac{1}{2} a^{2} \widehat{H}_{\lambda \lambda} \\
& -\frac{1}{2} a^{2}\left(1-\rho^{2}\right) \frac{\widehat{H}_{\lambda z}^{2}}{\widehat{H}_{z z}}+a \rho k s^{\beta+1} \widehat{H}_{s \lambda}+\frac{(\mu-r)^{2} a \rho z^{2}}{2 k^{2} s^{2 \beta}} \widehat{H}_{z z} \\
& -(\mu-r) s z \widehat{H}_{s z}-\frac{(\mu-r) a \rho z}{k s^{\beta}} \widehat{H}_{\lambda z}=0 .
\end{aligned}
$$

Differentiating $g(t, s, \lambda, z)=\widehat{H}_{z}$ on both sides with respect to $t, s, \lambda$, and $z$, we have the following first order and second order partial derivatives:

$$
\begin{gathered}
\widehat{H}_{t z}=-g_{t}, \quad \widehat{H}_{s z}=-g_{s}, \quad \widehat{H}_{s s z}=-g_{s s}, \\
\widehat{H}_{z z}=-g_{z}, \quad \widehat{H}_{s \lambda z}=-g_{s \lambda}, \quad \widehat{H}_{z z z}=-g_{z z}, \\
\widehat{H}_{s z z}=-g_{s z}, \quad \widehat{H}_{\lambda z z}=-g_{\lambda z}, \quad \widehat{H}_{\lambda z}=-g_{\lambda}, \\
\widehat{H}_{\lambda \lambda z}=-g_{\lambda \lambda}, \quad \frac{\widehat{H}_{\lambda z}^{2}}{\widehat{H}_{z z}}=-\frac{g_{\lambda}^{2}}{g_{z}} .
\end{gathered}
$$


Therefore, differentiating (24) for $\widehat{H}$ with respect to $z$ and using the above derivatives, we derive

$$
\begin{aligned}
g_{t} & +r s g_{s}-r g+\lambda+\left[\frac{(\mu-r)^{2}}{k^{2} s^{2 \beta}}-r\right] z g_{z}+b g_{\lambda} \\
& +\frac{1}{2} k^{2} s^{2 \beta+2} g_{s s}+\frac{1}{2} a^{2} g_{\lambda \lambda}+a \rho k s^{\beta+1} g_{s \lambda} \\
& -(\mu-r) z s g_{s z}-\frac{(\mu-r) a \rho}{k s^{\beta}}\left[g_{\lambda}+z g_{\lambda z}\right] \\
& +\frac{1}{2} a^{2} \frac{g_{\lambda}^{2} g_{z z}-2 g_{\lambda} g_{z} g_{\lambda z}}{g_{z}^{2}}=0 .
\end{aligned}
$$
as

Thus, the optimal strategy (12) denoted by $g$ is rewritten

$$
\pi^{*}=\frac{k^{2} s^{2 \beta+1} g_{s}-(\mu-r) z g_{z}+a \rho k s^{\beta} g_{\lambda}}{g k^{2} s^{2 \beta}} .
$$

We solve (26) to obtain the dual variable $g$. Then, replacing $g$ into (27) we obtain the optimal strategy.

\section{An Approximation Solution for \\ CARA Utility}

Consider the CARA utility

$$
U(v)=-\frac{1}{q} e^{-q v}, \quad q>0,
$$

where $q$ is the coefficient of absolute risk aversions. We have

$$
g(T, s, \lambda, z)=-\frac{1}{q} \ln z .
$$

We try to find a solution for (26) in the following form:

$$
\begin{array}{r}
g(t, s, \lambda, z)=-\frac{1}{q}[m(t)(\ln z+h(t, y, \lambda))]+n(t), \\
y=s^{-2 \beta} .
\end{array}
$$

Then

$$
\begin{gathered}
g_{t}=-\frac{1}{q}\left[m_{t}(\ln z+h(t, y, \lambda))+m(t) h_{t}\right]+n_{t}, \\
g_{s}=\frac{2 \beta}{q} s^{-2 \beta-1} m(t) h_{y}, \\
g_{z}=-\frac{m(t)}{q z}, \quad g_{\lambda}=-\frac{m(t) h_{\lambda}}{q}, \\
g_{\lambda \lambda}=-\frac{m(t) h_{\lambda \lambda}}{q}, \quad g_{z z}=\frac{m(t)}{q z^{2}}, \\
g_{s s}=-\frac{m(t)}{q}\left[4 \beta^{2} s^{-4 \beta-2} h_{y y}+2 \beta(2 \beta+1) s^{-2 \beta-2} h_{y}\right], \\
g_{s z}=g_{\lambda z}=0, \\
g_{s \lambda}=\frac{2 \beta}{q} s^{-2 \beta-1} m(t) h_{y \lambda} .
\end{gathered}
$$

Substituting the above derivatives into (26), we obtain

$$
\begin{aligned}
& {\left[m_{t}-r m\right] \ln z+\left[r n-n_{t}-\frac{r m}{q}\right] q} \\
& +l_{0} h+l_{1} h+l_{2} h \\
& -\frac{1}{2} a^{2}\left(1-\rho^{2}\right) m h_{\lambda}^{2}=0,
\end{aligned}
$$

where

$$
\begin{aligned}
l_{0} h= & -b q+b m h_{\lambda}+\frac{1}{2 m} a^{2}\left(1-\rho^{2}\right) q^{2} \\
& +a^{2}\left(1-\rho^{2}\right) q h_{\lambda}, \\
l_{1} h= & m_{t} h+m h_{t}-2 \beta \mu y m h_{y}-r m h \\
& +k^{2} m\left(2 \beta^{2} y h_{y y}+\beta(2 \beta+1) h_{y}\right) \\
& +2 \beta(\mu-r) y m h_{y}, \\
l_{2} h= & -2 a \rho m \beta k \sqrt{y} h_{y \lambda} \\
& +\frac{1}{k} a \rho(\mu-r) \sqrt{y} q \\
& -\frac{1}{k} a \rho(\mu-r) m \sqrt{y} h .
\end{aligned}
$$

We decompose (32) into the three equations

$$
\begin{gathered}
m_{t}-r m=0, \\
n_{t}-r n-\frac{r m}{q}, \\
\left(l_{0}+l_{1}+l_{2}\right) h-\frac{1}{2} a^{2}\left(1-\rho^{2}\right) m h_{\lambda}^{2}=0 .
\end{gathered}
$$

We state that $m$ and $n$ are found from (34) and (35). We know that (36) is a nonlinear partial differential equation, which has not a general solution.

In order to find solutions of (36), we let $\lambda$ vary slowly and a small parameter $\varepsilon(0<\varepsilon \ll 1)$ satisfy

$$
d \lambda_{t}^{\varepsilon}=\varepsilon b(\lambda)+\sqrt{\varepsilon} a(\lambda) d z(t) .
$$

Substituting (37) into (36), namely, replacing $b$ and $a$ with $\varepsilon b$ and $\sqrt{\varepsilon} a$, respectively in (36), we know that the corresponding $h^{\varepsilon}$ in (36) is rewritten in the form

$$
\left(\varepsilon l_{0}+l_{1}+\sqrt{\varepsilon} l_{2}\right) h^{\varepsilon}-\frac{1}{2} \varepsilon a^{2}\left(1-\rho^{2}\right) m\left(h_{\lambda}^{\varepsilon}\right)^{2}=0 .
$$

Theorem 2. If a solution to (38) takes in the form

$$
\begin{aligned}
h^{\varepsilon}(t, y, \lambda)= & h^{(0)}(t, y, \lambda)+\sqrt{\varepsilon} h^{(1)}(t, y, \lambda) \\
& +\varepsilon h^{(2)}(t, y, \lambda)+\cdots+\varepsilon^{n / 2} h^{(n)}(t, y, \lambda),
\end{aligned}
$$

then the approximation solutions to (36) in the slow-fluctuating regime are $h^{(0)}(t, y, \lambda), h^{(1)}(t, y, \lambda)$, and $h^{(2)}(t, y, \lambda)$. Moreover,

$$
h^{(0)}(t, y, \lambda)=e^{-\int p_{0} d t}\left[c_{0}+\int\left(q_{0} e^{-\int p_{0} d t}\right) d t\right],
$$


where $p_{0}=\left(m_{t}-r m\right) / m, q_{0}=k^{2} \beta(2 \beta+1) e^{-((m(\mu-r)+\mu) / k m \beta) y}$;

$$
h^{(1)}(t, y, \lambda)=e^{-\int p_{1} d t}\left[c_{1}+\int\left(q_{1} e^{-\int p_{1} d t}\right) d t\right]
$$

where $p_{1}=\left(m_{t}-r m\right) / m, q_{1}=k^{2} \beta(2 \beta+1) e^{-(((\mu-r) m+\mu) / k m \beta) y}+$ $(\mu-r) a \rho q \sqrt{y} / \mathrm{km}$

$$
h^{(2)}(t, y, \lambda)=e^{-\int p_{2} d t}\left[c_{2}+\int\left(q_{2} e^{-\int p_{2} d t}\right) d t\right]
$$

where $p_{2}=\left(m_{t}-r m\right) / m, q_{2}=k^{2} \beta(2 \beta+1) e^{((\mu-m(\mu-r)) / m k) y}+$ $(\mu-r) a \rho q \sqrt{y} / m k-b q / m-\left(1 / 2 m^{2}\right) a^{2}\left(1-\rho^{2}\right) q^{2}$.

Proof. Substituting (39) into (38), we derive

$$
\begin{aligned}
& l_{1} h^{(0)}+ \sqrt{\varepsilon}\left(l_{1} h^{(1)}+l_{2} h^{(0)}\right) \\
&+\varepsilon\left[l_{0} h^{(0)}+l_{1} h^{(2)}+l_{2} h^{(1)}\right. \\
&\left.-\frac{1}{2} a^{2}\left(1-\rho^{2}\right) m\left(h_{\lambda}^{(0)}\right)^{2}\right]=0 .
\end{aligned}
$$

Collecting the same order of the terms, we obtain the following three equations.

Zero-order term:

$$
l_{1} h^{(0)}=0, \quad \text { with } h^{(0)}(T, y, \lambda)=0
$$

term of order $\sqrt{\varepsilon}$ :

$$
l_{1} h^{(1)}+l_{2} h^{(0)}=0, \quad \text { with } h^{(1)}(T, y, \lambda)=0 ;
$$

term of order $\varepsilon$

$$
\begin{aligned}
l_{0} h^{(0)}+l_{1} h^{(2)}+l_{2} h^{(1)}-\frac{1}{2} a^{2}\left(1-\rho^{2}\right) m\left(h_{\lambda}^{(0)}\right)^{2} & =0, \\
\text { with } h^{(2)}(T, y, \lambda) & =0 .
\end{aligned}
$$

Considering the boundary condition, we will obtain solutions to $(44)-(46)$.

(i) From (44), we have

$$
\begin{aligned}
& m_{t} h^{(0)}+m h_{t}^{(0)}-2 \beta \mu y h_{y}^{(0)}-r m h^{(0)} \\
& +m k^{2}\left[2 \beta^{2} y h_{y y}^{(0)}+\beta(2 \beta+1) h_{y}^{(0)}\right] \\
& +2 \beta m y(\mu-r) h_{y}^{(0)}=0 .
\end{aligned}
$$

We decompose (47) into two equations

$$
\begin{aligned}
& y\left[-2 \beta \mu h_{y}^{(0)}+2 m k^{2} \beta^{2} h_{y y}^{(0)}+2 \beta m(\mu-r) h_{y}^{(0)}\right]=0, \\
& m_{t} h^{(0)}+m h_{t}^{(0)}-r m h^{(0)}+m k^{2} \beta(2 \beta+1) h_{y}^{(0)}=0 .
\end{aligned}
$$

From (48), we obtain $h_{y}^{(0)}$. It is

$$
\begin{gathered}
{\left[-2 \beta \mu h_{y}^{(0)}+2 \beta m(\mu-r) h_{y}^{(0)}\right]=-2 m k^{2} \beta^{2} h_{y y}^{(0)},} \\
\frac{\mu h_{y}^{(0)}-m(\mu-r) h_{y}^{(0)}}{m k^{2} \beta h_{y y}^{(0)}}=\frac{d h_{y}^{(0)}}{h_{y}^{(0)}}=d\left(\ln h_{y}^{(0)}\right), \\
h_{y}^{(0)}=e^{\left((\mu-m(\mu-r)) / m k^{2} \beta\right) y} .
\end{gathered}
$$

Putting (50) into (49), we obtain

$$
\begin{array}{r}
m_{t} h^{(0)}+m h_{t}^{(0)}-r m h^{(0)} \\
+m k^{2} \beta(2 \beta+1) e^{\left((\mu-m(\mu-r)) / m k^{2} \beta\right) y}=0, \\
h^{(0)}(t, y, \lambda)=e^{-\int p_{0} d t}\left[c_{0}+\int\left(q_{0} e^{-\int p_{0} d t}\right) d t\right],
\end{array}
$$

where $p_{0}=\left(m_{t}-r m\right) / m, q_{0}=k^{2} \beta(2 \beta+1) e^{-((m(\mu-r)+\mu) / k m \beta) y}$.

(ii) From (45), we have

$$
\begin{aligned}
& m_{t} h^{(1)}+m h_{t}^{(1)}-2 \beta \mu y m h_{y}^{(0)}-r m h^{(1)} \\
& \quad+m k^{2}\left[2 \beta^{2} y h_{y y}^{(1)}+\beta(2 \beta+1) h_{y}^{(1)}\right] \\
& \quad+2 \beta m y(\mu-r) h_{y}^{(1)}+\frac{1}{k}(\mu-r) a \rho q \sqrt{y}=0 .
\end{aligned}
$$

We decompose (52) into two equations

$$
\begin{gathered}
y\left[-2 \beta \mu h_{y}^{(1)}+2 m k^{2} \beta^{2} h_{y y}^{(1)}+2 \beta m(\mu-r) h_{y}^{(1)}\right]=0, \\
m_{t} h^{(1)}+m h_{t}^{(1)}-r m h^{(1)}+m k^{2} \beta(2 \beta+1) h_{y}^{(1)} \\
+\frac{1}{k}(\mu-r) a \rho q \sqrt{y}=0 .
\end{gathered}
$$

From (53), we obtain $h_{y}^{(1)}$. It is

$$
h_{y}^{(1)}=e^{((\mu-m(\mu-r)) / m k \beta) y} .
$$

Putting $h_{y}^{(1)}$ into (54), we obtain the solution

$$
h^{(1)}(t, y, \lambda)=e^{-\int p_{1} d t}\left[c_{1}+\int\left(q_{1} e^{-\int p_{1} d t}\right) d t\right],
$$

where $p_{1}=\left(m_{t}-r m\right) / m, q_{1}=k^{2} \beta(2 \beta+1) e^{-(((\mu-r) m+\mu) / k m \beta) y}+$ $(\mu-r) a \rho q \sqrt{y} / \mathrm{km}$.

(iii) From (46), we have

$$
\begin{gathered}
y\left[2 \beta \mu m h_{y}^{(2)}+2 m k^{2} \beta^{2} h_{y y}^{(2)}+2 \beta m(\mu-r) h_{y}^{(2)}\right] \\
+m h_{t}^{(2)}+\left(m_{t}-r m\right) h^{(2)}+m k^{2} \beta(2 \beta+1) h_{y}^{(2)} \\
+\frac{1}{k}(\mu-r) a \rho q \sqrt{y}-\frac{1}{2 m} a^{2}\left(1-\rho^{2}\right) q^{2}=0 .
\end{gathered}
$$

We decompose (57) into the following two equations

$$
\begin{aligned}
y\left[2 \beta \mu m h_{y}^{(2)}+2 m k^{2} \beta^{2} h_{y y}^{(2)}+2 \beta m(\mu-r) h_{y}^{(2)}\right] & =0, \\
m h_{t}^{(2)} & +\left(m_{t}-r m\right) h^{(2)}+m k^{2} \beta(2 \beta+1) h_{y}^{(2)} \\
+ & \frac{1}{k}(\mu-r) a \rho q \sqrt{y}-\frac{1}{2 m} a^{2}\left(1-\rho^{2}\right) q^{2}=0 .
\end{aligned}
$$


From (58), we obtain $h_{y}^{(2)}$. It is

$$
h_{y}^{(2)}=e^{((\mu-m(\mu-r)) / m k) y} .
$$

Putting $h_{y}^{(2)}$ into (59), we get the solution

$$
h^{(2)}(t, y, \lambda)=e^{-\int p_{2} d t}\left[c_{2}+\int\left(q_{2} e^{-\int p_{2} d t}\right) d t\right],
$$

where $p_{2}=\left(m_{t}-r m\right) / m, q_{2}=k^{2} \beta(2 \beta+1) e^{((\mu-m(\mu-r)) / m k) y}+$ $(\mu-r) a \rho q \sqrt{y} / m k-b q / m-\left(1 / 2 m^{2}\right) a^{2}\left(1-\rho^{2}\right) q^{2}$. Therefore, (51), (56), and (61) are the solutions to (44)-(46) which are the solutions to (36).

Considering

$$
\begin{gathered}
g=-\frac{\ln z}{q}, \quad z g_{z}=-\frac{m}{q}, \\
g_{s}=\frac{2}{q} m \beta s^{-2 \beta-1} h_{y}, \quad g_{\lambda}=-\frac{m}{q} h_{\lambda}, \\
m_{t}=r m, \quad g=v,
\end{gathered}
$$

from (27), (51), (56), and (61), we have

$$
\begin{gathered}
\pi_{t}^{*}=\frac{m(\mu-r)+k^{2} s^{2 \beta+1} 2 \beta s^{-2 \beta-1} m h_{y}-k s^{\beta} \rho a m h_{\lambda}}{q v k^{2} s^{2 \beta}} \\
=\frac{m}{q v k^{2} s^{2 \beta}}\left\{(\mu-r)+2 \beta k^{2} h_{y}-a \rho k s^{\beta} h_{\lambda}\right\} \\
=\frac{m}{q v k^{2} s^{2 \beta}}\left\{(\mu-r)+2 \beta k^{2}\left[h_{y}^{(0)}+\sqrt{\varepsilon} h_{y}^{(1)}+\varepsilon h_{y}^{(2)}\right]\right. \\
\quad-a \rho k s^{\beta}\left[h_{\lambda}^{(0)}+\sqrt{\varepsilon} h_{\lambda}^{(1)}+\varepsilon h_{\lambda}^{(2)}\right] \\
\left.+o\left(\varepsilon^{3 / 2}\right)\right\} \\
=\frac{m}{q v k^{2} s^{2 \beta}}\left\{(\mu-r)+2 \beta k^{2} h_{y}^{(0)}-a \rho k s^{\beta} h_{\lambda}^{(0)}\right. \\
+\sqrt{\varepsilon}\left(2 \beta k^{2} h_{y}^{(1)}-a \rho k s^{\beta} h_{\lambda}^{(1)}\right) \\
\left.+\varepsilon\left(2 \beta k^{2} h_{y}^{(2)}-a \rho k s^{\beta} h_{\lambda}^{(2)}\right)+o\left(\varepsilon^{3 / 2}\right)\right\} .
\end{gathered}
$$

When $\varepsilon \rightarrow 0$ and according to (50) and (51), we have

$$
\pi_{t}^{*}=\frac{m}{q v k^{2} s^{2 \beta}}\left\{(\mu-r)+2 \beta k^{2} e^{(\mu-m(\mu-r)) / m \beta k^{2} s^{2 \beta}}\right\} .
$$

\section{Conclusion}

In the optimal investment strategies, we consider personal wealth with consumption invested in risk-free asset and risky asset. We apply the $C E V$ model to the optimal personal portfolio and obtain the dual solution by using the Legendre transform and dual theory. Maximizing the expected utility and using the corresponding Hamilton-Jacobi-Bellman
$(H J B)$ equation, we obtain an approximation solution and the optimal investment strategies for the exponential utility function. The results obtained in this paper are only applicable for the exponential utility function. For other cases, it would extend this result for further research in our forthcoming paper.

\section{Conflict of Interests}

The authors declare that they have no conflict of interests.

\section{References}

[1] W. H. Fleming and T. Zariphopoulou, "An optimal investment/consumption model with borrowing," Mathematics of Operations Research, vol. 16, no. 4, pp. 802-822, 1991.

[2] J. Vila and T. Zariphopoulou, "Optimal consumption and portfolio choice with borrowing constraints," Journal of Economic Theory, vol. 77, no. 2, pp. 402-431, 1997.

[3] R. Yao and H. H. Zhang, "Optimal consumption and portfolio choices with risky housing and borrowing constraints," The Review of Financial Studies, vol. 18, no. 1, pp. 197-239, 2005.

[4] J. C. Cox and S. A. Ross, "The valuation of options for alternative stochastic processes," Journal of Financial Economics, vol. 3, no. 1-2, pp. 145-166, 1976.

[5] M. Schroder, "Computing the constant elasticity of variance option pricing formula," Journal of Finance, vol. 44, pp. 211-219, 1989.

[6] C. F. Lo, P. H. Yuen, and C. H. Hui, "Constant elasticity of variance option pricing model with time-dependent parameters," International Journal of Theoretical and Applied Finance, vol. 3, pp. 661-674, 2000.

[7] P. P. Boyle and Y. Tian, "Pricing lookback and barrier options under the CEV process," Journal of Financial and Quantitative Analysis, vol. 34, no. 2, pp. 241-264, 1999.

[8] D. Davydov and V. Linetsky, "Pricing and hedging pathdependent options under the CEV process," Management Science, vol. 47, no. 7, pp. 949-965, 2001.

[9] F. Black and M. Scholes, "The pricing of options and corporate liabilities," Journal of Political Economy, vol. 81, pp. 637-654, 1973.

[10] J. C. Cox, "The constant elasticity of variance option pricing model," Journal of Portfolio Management, vol. 22, pp. 16-17, 1996.

[11] J. Detemple and W. D. Tian, "The valuation of american options for a class of diffusion processes," Management Science, vol. 48, no. 7, pp. 917-937, 2002.

[12] C. Jones, "The dynamics of stochastic volatility: evidence from underlying and options markets," Journal of Econometrics, vol. 116, no. 1-2, pp. 181-224, 2003.

[13] J. Xiao, Z. Hong, and C. Qin, "The constant elasticity of variance (CEV) model and the Legendre transform-dual solution for annuity contracts," Financial Insurance: Mathematics and Economics, vol. 40, no. 2, pp. 302-310, 2007.

[14] J. Gao, "Optimal portfolios for DC pension plans under a CEV model," Insurance: Mathematics and Economics, vol. 44, no. 3, pp. 479-490, 2009.

[15] J. Gao, "An extended CEV model and the Legendre transformdual-asymptotic solutions for annuity contracts," Insurance: Mathematics \& Economics, vol. 46, no. 3, pp. 511-530, 2010. 
[16] M. Jonsson and R. Sircar, "Optimal investment problems and volatility homogenization approximations," Modern Methods in Scientific Computing and Applications NATO Science Series II, vol. 75, pp. 225-281, 2002.

[17] E. J. Jung and J. H. Kim, "Optimal investment strategies for the HARA utility under the constant elasticity of variance model," Insurance: Mathematics and Economics, vol. 51, no. 3, pp. 667673, 2012.

[18] D. Kramkov and W. Schachermayer, "The asymptotic elasticity of utility functions and optimal investment in incomplete markets," The Annals of Applied Probability, vol. 9, no. 3, pp. 904-950, 1999.

[19] J. C. Cox and C. Huang, "A variational problem arising in financial economics," Mathematics in Economics, vol. 20, no. 5, pp. 16-17, 1991. 


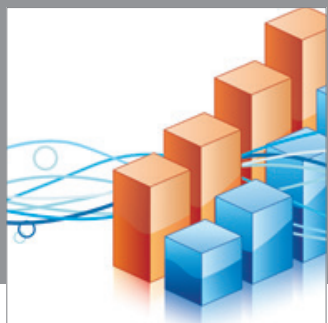

Advances in

Operations Research

mansans

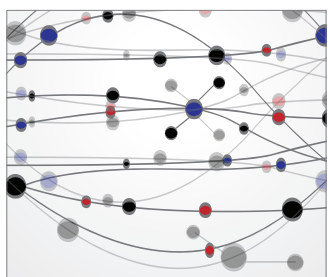

The Scientific World Journal
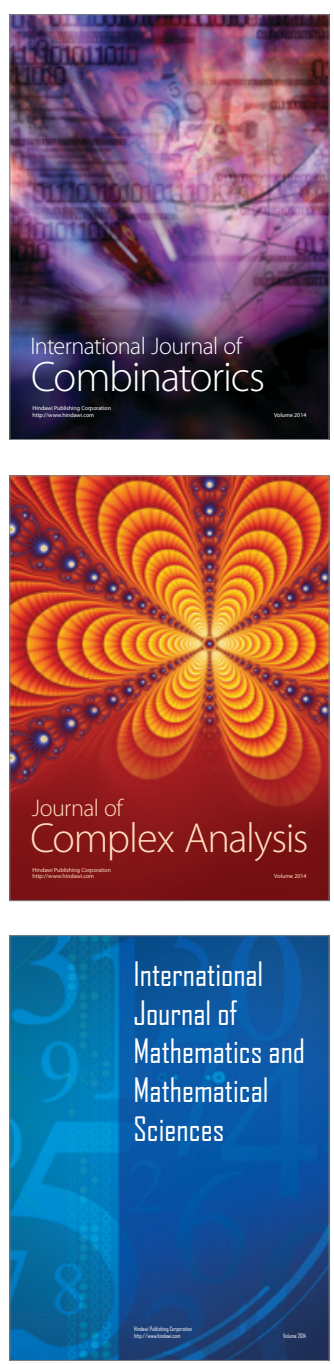
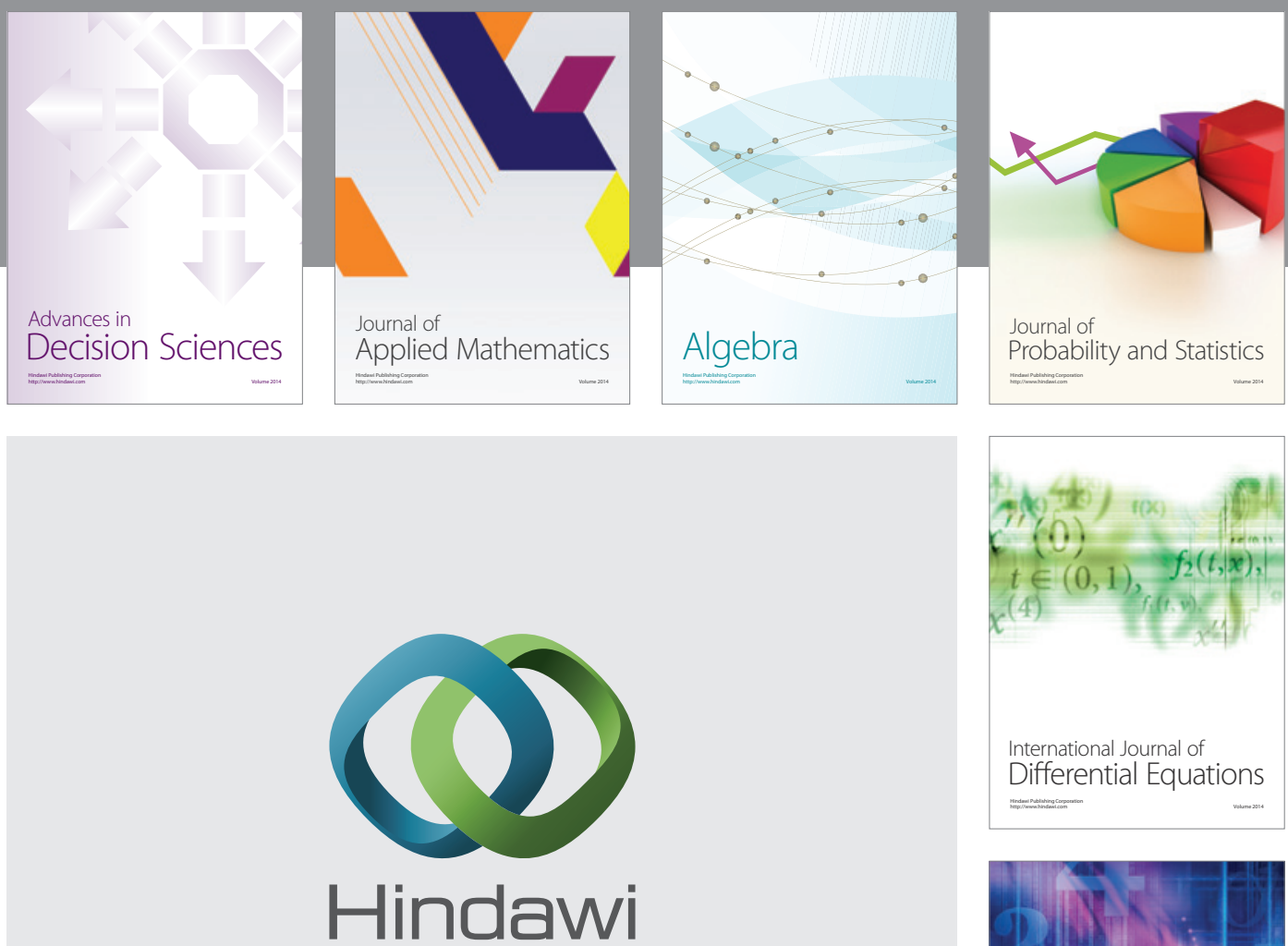

Submit your manuscripts at http://www.hindawi.com
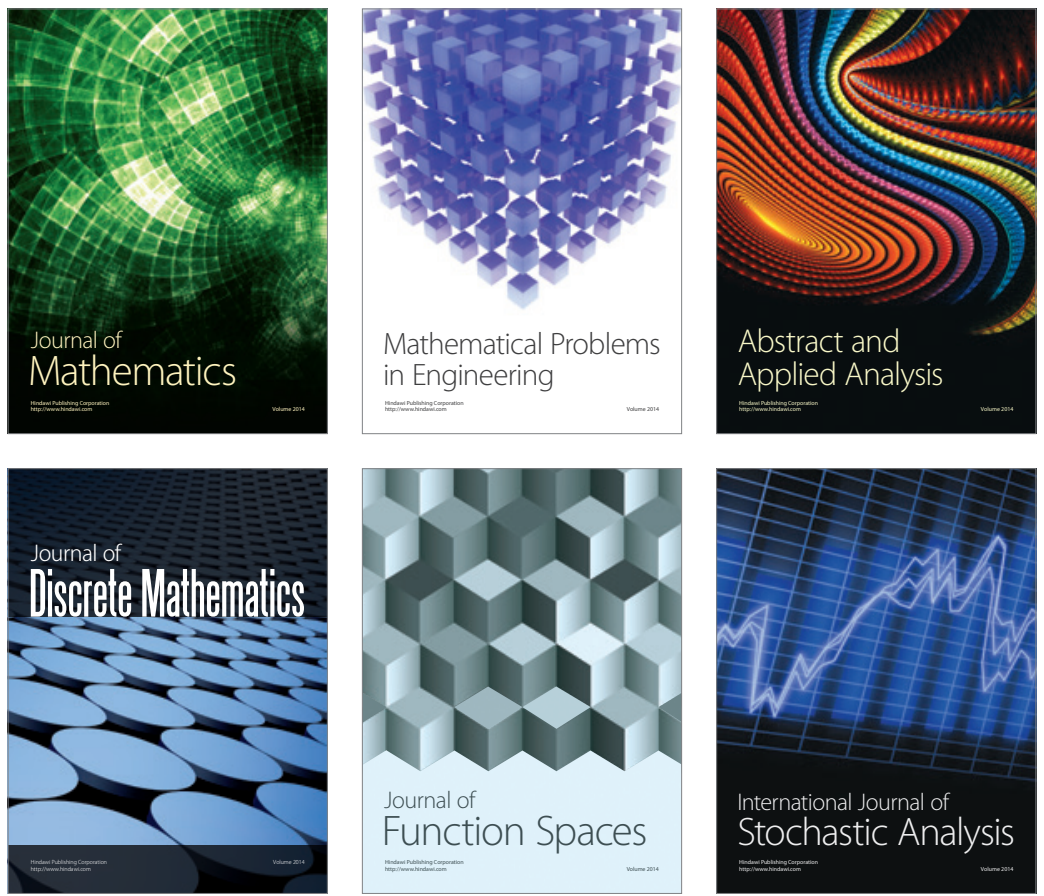

Journal of

Function Spaces

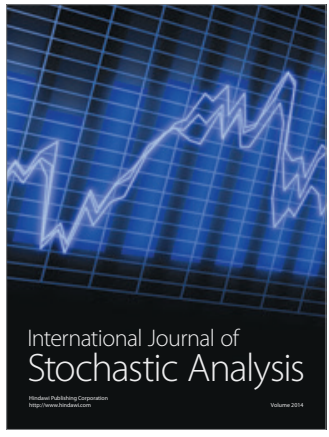

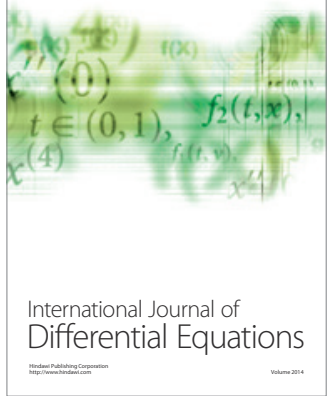
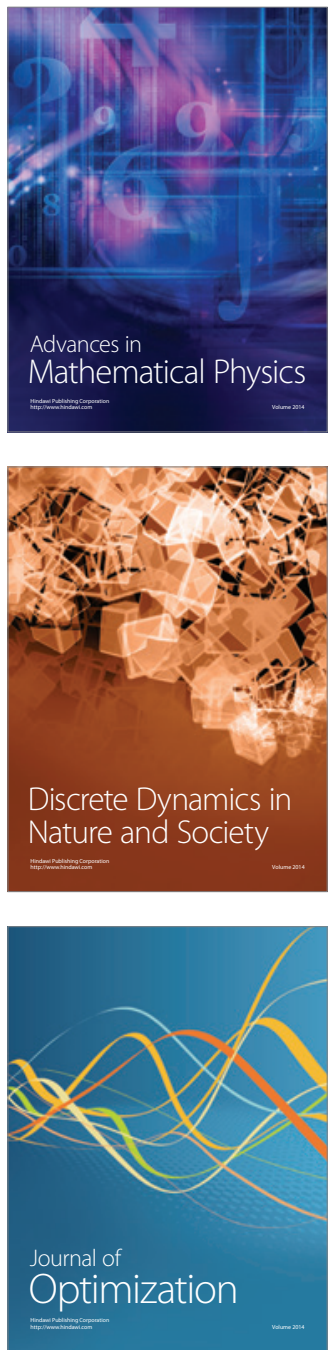